\title{
Atestación y Respuesta: Reflexión a Partir de una Fenomenología Hermenéutica de las Capacidades ${ }^{1}$
}

RESUMEN: El siguiente ensayo busca explorar la tesis según la cual en la atestación o reconocimiento de sí, el sí-mismo que está en juego está atestiguado primeramente de modo prospectivo. Atestiguarse es reconocerse no en sí, sino en un envío, en una conminación que resulta del encuentro con lo otro; de este modo, se puede afirmar que la identidad personal es ante todo una identidad de éxodo, es decir enviada. Para el análisis de estas tesis quisiéramos centrarnos en la fenomenología hermenéutica del hombre capaz que desarrolla Ricœur de modo explícito en Soi-même comme un autre, y que ya ha bosquejado desde Le volontaire et l'involontaire. Con ello interrogaremos en qué sentido las capacidades son un modo de ser respondiente del sí-mismo ante lo otro que lo envía, y luego interrogaremos, en los márgenes de la hermenéutica bíblica del autor, el alcance de lo que significa responder para el sí-mismo.

PALABRAS CLAVES: Sí-Mismo. Atestación. Capacidades. Llamada-Respuesta. Envío.

En Parcours de la reconnaissance, al comienzo de su estudio segundo sobre el reconocimiento de sí, Paul Ricœur propone una extensión de su hermenéutica de la atestación llevada a cabo en Soi-même comme un autre (1990), subrayando el vínculo entre el reconocimiento de sí y la atestación. $\mathrm{Si}$ bien nuestro autor reconoce que ambos conceptos pertenecen a familias lexicales diferentes, al mismo tiempo sostiene un parentesco que le permite incluso hablar de un "reconocimiento-atestación" (RICEER, 2004, p. 141). Tal como en Soi-même comme un autre, en su último libro publicado

\footnotetext{
${ }^{1}$ Este artículo ha sido redactado en el marco del Proyecto Fondecyt $\mathrm{N}^{\circ} 11100061$. El autor agradece a Olivier Abel y Catherine Goldenstein que le permitieron trabajar durante el mes de mayo de 2011 en la Biblioteca del Fonds Ricoeur, pudiendo consultar textos inéditos y artículos publicados del autor de difícil acceso. También expresa su agradecimiento al IMEC de Caen y a los herederos legales de Ricoeur que le permitieron consultar correspondencia privada del filósofo así como literatura secundaria también de difícil acceso. Esta estadía de investigación ha sido apoyada por el proyecto Fondecyt $\mathrm{N}^{\circ} 11100061$
}

${ }^{2}$ Universidad Alberto Hurtado. pamenam@uahurtado.cl 
en vida Ricœur establece un nexo fundamental e inquebrantable entre este "reconocimiento-atestación" y el homme capable, pues es en nuestras capacidades que nos atestiguamos y reconocemos como un sí-mismo. Así, lo que quisiera explorar en este ensayo son las siguientes tesis: si el "reconocimiento-atestación" está ligado estructuralmente con las capacidades que el hombre es capaz de desplegar y, con ello, de desplegarse a sí mismo en el mundo, su razón estriba en que las capacidades humanas son un modo de ser estructural del sí-mismo cuyo fundamento es la relación y la respuesta; en este sentido, es una ontología de la relación la que está a la base de la hermenéutica del homme capable, y que reemplaza en su función meta a la ontología del acto y de la potencia que Ricœur defiende en el estudio décimo de Soi-même comme un autre (Cf. VALLÉ, 2010). Por lo demás, como consecuencia del vínculo estrecho entre reconocimiento-atestación y capacidades, se puede desprender una identidad retro y prospectiva, según la cual el sí-mismo, lejos de ser un dato, es una tarea por conquistar sin que nunca llegue el tiempo para decir "[...] hasta aquí llego, ya he realizado mi labor" (Cf. MICHEL, 2008, p. 72). Un sí capaz, por estas razones, es un sí-mismo inimaginable para sí: atestiguarse en las capacidades es reconocerse en una identidad de éxodo enviada hacia un porvenir impredecible para el sí (cf. HOUSSET, 2008).

En segundo lugar, una hermenéutica de las capacidades como estructura relacional y responsiva del sí-mismo pone el acento en los diversos modos que tiene el sí-mismo para decir "sí", para afirmar y afirmarse. En este sentido, el "reconocimiento-atestación" se inscribe en una ontología de la afirmación originaria y del consentimiento. El sí-mismo consiente su condición y la donación de sí en su doble acepción: donación dada al sí y que da al sí, por una parte, y donación para elegirse y afirmarse prospectivamente. En este sentido, la paciencia y el consentimiento son dos modos ejemplares que tensionan al sujeto entre la herencia y la invención, y hacen de la vehemencia ontológica un lugar insigne para pensar su atestiguación en sus capacidades y su reconocimiento como un inimaginable para sí, como aquel que se espera en lo inesperado de sí (cf. ROMANO, 2011).

En tercer lugar, quisiera subrayar un énfasis: si las capacidades implican una reflexividad a partir de la que el sí-mismo se atestigua y reconoce, entonces es posible retrotraer la fenomenología hermenéutica del hombre capaz hacia una hermenéutica de la llamada y la respuesta que fue desarrollada de modo explícito en el libro póstumo de Ricœur titulado Amour et justice (2008), y que reúne dos de las conferencias de las Gifford Lectures que Ricœur suprimió 
de la versión final de Soi-même comme un autre. Se trata, por cierto, de una hermenéutica bíblica que tiene su asidero en una hermenéutica reflexiva amplia que comprende tanto la de los símbolos como la de los textos, y que fue desplegada a partir de La symbolique du mal (1960), y desarrollada luego en Le conflit des interprétations (1969), Temps et récit (1983-1985) y Du texte à l'action (1986). Con el recurso a esta hermenéutica reflexiva, quisiera explorar una capacidad específica, la de responder a un llamado, a una conminación, a partir de la que el sí-mismo se configura y reconoce. El interés de este último punto consiste en la posibilidad de enfatizar el envío al que nos conmina el reconocimiento-atestación que se deja comprender en las respuestas que ofrecemos a los múltiples llamados a los que atendemos. La responsividad, entendida ésta como modo de ser del sí-mismo, supone que la atestación se da o se hace no del lado del sí, sino de la alteridad. Las respuestas que somos y que damos nos atestiguan prospectivamente en un porvenir y no meramente en un presente, ni retrospectivamente en un pasado: no es la memoria el mayor signo de la identidad personal, sino la paciencia, que es un punto relevante que quisiera intentar mostrar.

1 En una entrevista realizada por G. Jarczyck, Ricœur establece un vínculo estricto entre capacidad y humanidad:

Es la humanidad, afirma nuestro autor, lo que llamo "Sí-mismo" en definitiva, la cualidad humana, el hecho de poder considerarse como el autor de sus propios actos, como siendo capaz de acciones intencionales, de iniciativas que cambian realmente el curso de las cosas, como pudiendo situarse en un relato de vida, como siendo a la vez el narrador y el personaje de su propia historia. Es esa humanidad, no en el sentido extensivo del conjunto de hombres, sino que intensivo de la cualidad humana: lo que hace que un hombre sea un hombre. He ligado por consiguiente esta noción de humanidad, continúa Ricœur, con la capacidad reflexiva fundamental de designarse a sí mismo como aquel que habla, como aquel que actúa, como aquel que narra, "se narra" y como aquel que se siente responsable y a quien las consecuencias de sus actos pueden serle imputadas. (RICEUR, 1991, p. 229).

Las capacidades, tal como las comprende nuestro autor en este texto, son el lugar para la explicitación del sí: atestiguarse y reconocerse en ellas es, por consiguiente, explicitarse en el despliegue de los poderes a partir de los cuales el ser humano se abre y vuelve hacia el mundo y los otros, lo que 
supone sin embargo una explicitación también del mundo. Su comprensión y explicación aporta recursos para el retorno hacia sí tras el éxodo de este sujeto intencional hacia las cosas mismas.

De este texto es preciso destacar algunas consideraciones muy relevantes para el propósito de este ensayo. En primer lugar, el lugar privilegiado que nuestro autor le otorga a la noción de reflexividad, que no puede ser comprendida a su vez sin vínculo con la intencionalidad. La reflexividad, como un poder de retorno sobre sí, se "sostiene" en el movimiento intencional como salida fuera de sí, hacia las cosas y al mundo. La intencionalidad, que no queda restringida a la vida de la conciencia teórica sino que es ampliada a la esfera de la praxis humana, carga además con una opacidad que impide que el sujeto se comprenda directa e intuitivamente a sí mismo. En este sentido, ser capaz de volver la atención hacia las cosas mismas es también poder perderse en ellas, entre ellas, por las acciones que realizamos y que intencionan cambios y transformaciones del mundo de la vida. La intencionalidad es, de este modo, un movimiento que proyecta al sujeto fuera de sus goznes pero que no lo deja indemne. En este sentido, se podría afirmar que el movimiento descrito por Ricour es tanto intencional como transitivo, aunque este último término no le sea propio. Pues, el sujeto que quiere, por ejemplo y para tomar el caso de Le volontaire et l'involontaire, no solo proyecta una intención y un querer, sino que se proyecta a sí mismo hacia lo querido. Hay intencionalidad, el proyecto es un claro ejemplo de ello, pero también hay transitividad, pues finalmente no podría querer algo sin que acoja o reciba motivos para actuar, deseos, necesidades, y toda una suma de involuntarios que por un lado dan impulso a mi querer y por otro le resisten. Para el sujeto querer implica, por tanto, un movimiento intencional así como una transitividad a partir de la que el sí-mismo se reconoce al encuentro con una alteridad que no puede ser suprimida, sino tan solo resistida o consentida. Lo involuntario, en este sentido, puede ser resistido, como pasa con el esfuerzo con relación a la fatiga, pero finalmente esta última nunca es vencida del todo, sino solo parcialmente; al final del día no podemos dejar de caer rendidos por el cansancio.

De este modo, la reflexividad supone un movimiento intencional pero también una transitividad que en conjunto son la fuente de la opacidad del símismo; pues, finalmente, estar vuelto hacia afuera de sí, poseer una identidad de éxodo según la expresión de Emmanuel Housset (2008), supone ser capaz de hacer el encuentro con la alteridad como una instancia indomeñable en la que estamos, sin embargo, implicados. Así, si la reflexividad comprende un 
movimiento de salida y otro de retorno, también supone una implicación del sujeto con lo otro de sí. Hay opacidad porque el sí-mismo no es plenamente "por sî", vale decir no es totalmente ni autónomo ni autotélico, sino que también es, en buena parte, "por otro", es decir "heterónomo" (Cf. HOUSSET, 2010) - aunque esta no es una expresión del gusto de Ricœur me parece que aclara muy bien el movimiento de la reflexividad -. La reflexividad, en este sentido, es una capacidad a partir de la que el sí se explicita en un largo movimiento de ida y de vuelta, por el que se implica, se embrolla y se reconoce en la alteridad. La opacidad que aporta la reflexividad al sí se explica entonces por la toma de distancia que debe asumir el sujeto consigo mismo, pues el encuentro con lo otro de sí, con la alteridad (del cuerpo, lo involuntario relativo y absoluto, del otro y del mundo, etc.), supone no solo una imbricación con ella, sino también un intento de apropiación de sí en vínculo con esta alteridad que, a su vez, es constituyente del modo de ser del sí-mismo. Ser sí-mismo no es ser otro, pero sí ser "como" otro, donde el "como" marca el carácter implicante de la alteridad como también una distancia insalvable del todo: de este modo, el sí no puede tomar suficiente distancia con lo otro de sí, pero tampoco puede apropiárselo del todo.

Se comprende mejor, entonces, la complejidad del acto de autodesignación a partir del que Ricœur define la reflexividad, pues finalmente el sujeto que se nombra a sí mismo no tiene certeza última ni apodíctica de aquello que nombra cuando dice "yo". De este modo, el pronombre reflexivo "sí-mismo" tiene ventaja sobre el "yo", pues es el modo que tiene el sujeto de llamarse en la implicación con la alteridad, de reconocerse "como" otro, y por tanto de comprenderse autónomo y heterónomo a la vez.

2. Por otra parte, si las capacidades son el lugar de la explicitación de sí, al mismo tiempo, éste se atestigua en ellas como un "quién" que cree que puede, que confía en sus poderes a partir de los que se lanza al mundo. El tipo de confianza que supone la atestación no tiene como fundamento ninguna apodicticidad ni certidumbre última; se trata tan solo de una creencia, de una convicción, si se quiere, de que el "quién" que soy se despliega en el "yo puedo". Las capacidades no son solo el lugar de la explicitación de sí, sino la instancia a partir de la que el sí-mismo se esfuerza y persiste en ser un "quién" capaz de asumirse, de reencontrarse, de reconquistarse en el yo creo que "puedo": es así que Ricœur (1950, p. 56) declara en Le volontaire et l'involontaire que el "[...] yo es en sus actos". Si esta posición parece requerir matices, sobre todo a partir 
de los estudios sobre el perdón, nuestro autor mantiene intacta la convicción de que el sujeto capaz lo es, primeramente, de reivindicar un curso de acción (RICEUR, 1936). En este sentido, el yo que puede es remitido a la capacidad de apropiarse a sí mismo en sus acciones, en sus proyectos, en su querer, en todos los movimientos que animan su vida intencional; lo que significa que es capaz de volver sobre sí, de reconocerse en un curso de acción propuesto previamente y, a pesar de los cambios de convicciones, de valoraciones, de posiciones que agregan distancia y opacidad al reconocimiento, aún puede designarse como autor, incluso a su pesar. ¿No hay aquí, entonces, en estos textos tempranos prehermenéuticos, una prefiguración de lo que Ricœur llamará en su obra tardía, una ipseidad definida como un modo de ser del símismo abierto a la alteridad y que nos aporta comprensión según el modelo de la promesa? Es decir, un sí que es capaz de reivindicar un curso de acción, también es capaz de persistir en sus promesas, en sus proyectos y en los lineamientos que propone para su acción; es un sí, por ende, que no solo se reconoce en la acción promovida y en sus efectos, sino que se atestigua en la promesa que toda acción libra. La atestación y reconocimiento de sí, en este sentido, no reconquista ningún sujeto sustancial, ninguna identidad del tipo de la mismidad, sino a un sí-mismo que es capaz de persistir en las promesas que ofrece y dona. Lo que supone que el sí que promete confía en su persistencia, inclusive si no puede saber cuál será el sí que debe reivindicar un curso de acción prometido, un proyecto intencionado, querido y ejecutado: el sí que se asume reconociéndose capaz de reivindicación, es un sujeto inimaginable; por ello la atestación depende de una confianza, de una creencia que en las capacidades por las que obramos aún hay un quién que puedo reconocer.

Por otra parte, es necesario ligar los análisis precedentes del hombre capaz de autodesignarse como el agente, el narrador, el sujeto responsable, etc., que Ricœur ha llamado reflexión. Pues, lo que está en juego aquí no es solo la opacidad, sino la futuridad a la que ésta envía al sujeto reflexivo: de este modo, se puede afirmar que el sujeto capaz de perderse en el mundo, en las mediaciones simbólicas y culturales, en las obras, y capaz de reconquistarse a su vez, queda implicado en una espera de lo inesperable o, dicho de otro modo, él mismo se vuelve para sí un sujeto inimaginable, indeterminable, inacabable en términos comprensivos. Para entender esto es preciso insistir con la mayor fuerza posible en el hecho de que el ser humano, en su ser, está expuesto a la contingencia de las cosas, o de otro modo, al contacto con las cosas, con el mundo, con el otro, que es lo que hemos subrayado al poner en relación a la intencionalidad con la transitividad. Dicho contacto o encuentro con la 
alteridad implica por parte del sí-mismo un estremecimiento, una experiencia (expereri) en el sentido fuerte del término, a saber un aventuramiento, un riesgo de sí. Este sentido de "experiencia", que ha sido destacado por Henri Maldiney (2007), Claude Romano (1998), Jean Greisch (2003/4) y Claude Gens (2009) entre otros, nos permite pensar la dimensión pasible del sujeto así como su proyectarse en el mundo: acentúa la acogida u hospitalidad como modo de ser del sí-mismo por la que para el sujeto abrirse al mundo implica una auto y heteroafección; pero también pone el acento en la capacidad que tiene el sí-mismo de abrirse hacia las cosas, de volverse hacia el mundo asumiendo el riesgo de la distancia entre sí y sí mismo, y por tanto de la opacidad. Es en este sentido que se puede comprender la siguiente afirmación de Romano: “[...] experienciar es dejarse alcanzar por lo que viene a nosotros [...] La experiencia es una travesía, lo que supone una distancia e intervalo y un franqueamiento, de sí a sí, por lo cual solo podemos acoger lo que nos adviene, adviniendo a nosotros mismos como otro" (1998, p. 195; Cf. MENA, 2011, p. 681682). Que la experiencia sea una travesía significa que no es pura acogida, pues recibir implica un movimiento intencional, una salida fuera de sí, un volverse hacia algo que yo no soy, pero que ya ha arribado en mí, tal vez sin mí, estremeciendo al sujeto en sus capacidades, proveyéndole de una diferencia que jamás puede ser reducida ni suprimida. Ciertamente, esta noción de experiencia, que depende de una fenomenología del acontecimiento (Cf. ROMANO, 1998; MALDINEY, 2007), desafía a la subjetividad y su poder de retomarse a sí misma, de reconocerse como la misma, abriendo en ella una diferencia fundamental e irreductible; ‘ante los acontecimientos que nos suceden, aquellos que vienen con una fuerza trastocante de sentido, podemos reconocernos como los mismos?

Pero, podemos conservar sin embargo una enseñanza: lo que hace del sujeto un sí-mismo es su apertura y relación hacia lo otro de sí. Lo interesante de esto no es solo esta apertura pasible y relacional, sino la persistencia en la implicación con la alteridad; el sí-mismo es aquel que insiste en esta imbricación, es, por tanto, un sujeto paciente en el sentido más fuerte del término; ni meramente pasivo, ni meramente activo, pero resueltamente en vínculo con lo otro de sí. Porque el ser del sí-mismo, podríamos decir su ipseidad extendiendo tal vez el uso que le da Ricœur a este término, pone en juego su ser en la paciencia y en la constancia que le es propia, es que su atestación y reconocimiento no se sostiene en un ego puro y apodíctico, sino en un sujeto aventurado hacia un porvenir inanticipable que, en estricto rigor, no puede esperarse porque no termina de darse ni de recibirse. El sí es por tanto 
"por sî" y "por otro", es original pero no originario, reivindica sus acciones y sus promesas y con ello a un "quien" que es capaz de constar y persistir.

Quisiera dejar hasta aquí el problema del fundamento del sí, pues esta cuestión será retomada luego en este texto. A continuación, quisiera subrayar de modo aún más explícito la dimensión relacional del "ser capaz" junto con su carácter responsivo.

3. En primer lugar, las capacidades como estructura del ser del símismo, su ipseidad, solo pueden ser pensadas en su relación estrecha con la experiencia. De este modo, se puede afirmar que éstas, las capacidades, son "[...] aptitudes para la experiencia" (LACOSTE, 2011, p. 328) o dicho de otra forma, la experiencia que hacemos del mundo y del otro dependen de un "yo puedo capaz" que por sus poderes se abre e intenciona a las cosas mismas de un modo específico, en la medida que las capacidades nos donan un porvenir y unos posibles sin los que no podríamos entrar al mundo. Es así que Jean-Yves Lacoste afirma, en consonancia por lo demás con el último trabajo de Romano (2011 a; 2011 b), que "[...] toda experiencia está fundada en una aptitud global a la experiencia que la desborda” (ROMANO, 2011, p. 328).

Las capacidades en las que nos atestiguamos nos abren, por consiguiente, a un porvenir y a unos posibles que podemos apropiarnos y proyectar y proyectarnos con ellos. Consideremos el siguiente ejemplo: aprender a tocar el piano, y por tanto adquirir hábitos determinados de interpretación, llenar nuestras manos de una memoria cinestésica, etc., en suma, volverse capaz de tocar una melodía en el piano implica darse posibilidades que hasta el momento no constituían ni mi horizonte perceptivo, ni cinestésico, ni práctico. Antes de aprender a tocar el piano no me era posible interpretar el Rondo alla turca de Mozart. Asumir y asumirse en una capacidad es, de este modo, abrirse a una serie de posibilidades que determinan nuestra experiencia tanto del mundo como de nosotros mismos. De aquí en adelante me puedo reconocer en una serie de actividades, de hábitos, y de actitudes y comportamientos que me eran desconocidos en "primera persona"; pero al mismo tiempo esta relación con los posibles, que ahora puedo proyectar o que puedo encontrar, hace del sí-mismo que soy un sujeto no constituido del todo, no finiquitado en su ser, sino uno que tiene que asumirse y atestiguarse en aquellos posibles que pueden figurarme de otro modo, que pueden darme a mí mismo otro rostro: en cierto sentido, me vuelvo para mí inimaginable, al menos en lo que espero 
de mí, en cuanto un "quién” que es capaz siempre de confrontarse a sí mismo a través de la asunción de otros posibles. La atestación y reconocimiento de sí no recaen tan solo en la capacidad de la memoria, sino también en la espera, lo que obliga al sí-mismo a confiar que en su "ser capaz" se mantiene aún un "quién" que puede responder de sí y del modo como se apropia esos posibles nuevos a los que se ha abierto en sus capacidades. Cuando Ricœur afirma, por tanto, que el hombre se atestigua y reconoce en sus poderes, lo que está acentuando respecto de la ipseidad, como modo de ser abierto, relacional y respondiente del sí-mismo, es que ésta es primeramente un "poder-ser" que, lejos de plantear un sujeto transparente para sí, supone un sujeto haciéndose, confrontándose consigo mismo, sus capacidades y sus posibles. La ipseidad es, en este sentido, un modo de ser que tiene su fundamento en los poderes a partir de los que el hombre se abre a un mundo y al mundo. Que Ricœur considere que la promesa es el modelo ejemplar de la ipseidad no es erróneo, en la medida que se la considere un poder ser a partir del que me abro a una serie de posibles en los que puedo persistir; así también, la promesa es la posibilidad de entrar en relación con el otro a través del compromiso, lo que supone también una fidelidad a la palabra ofrecida. La promesa está cargada de posibles, pues siendo una capacidad, ésta me abre al mundo singularmente a partir de las experiencias de la persistencia y la constancia, de la fidelidad y del compromiso. Yo me puedo reconocer en las promesas que ofrezco, inclusive si ya no me considero el mismo sujeto que las ha pronunciado. Pero, hay aquí otro punto relevante, pues tal como lo afirma Lacoste, todo "[...] poder-ser critica siempre lo que somos" (LACOSTE, 2011, p. 329)Que el hombre capaz se atestigue en sus poderes a partir de los que se abre al mundo, a los otros y a lo otro de sí, significa también que en sus capacidades no solo entra en relación con..., sino que cada poder es la ocasión, al menos, para la contestación de lo que soy en la medida que "ser capaz" es darse y disponerse a posibilidades cada vez nuevas que asumimos o podemos apropiarnos. Así, reconocerse en los poderes que somos capaces de desplegar es atestiguar un sí que, en su ser, responde del sí que se despliega, patentizando un hiato, un quiebre en el hombre capaz. El fundamento de la contestación que son las capacidades, se halla precisamente en la experiencia que hacemos de nosotros mismos como sujetos capaces: pues, en última instancia, el poder-ser que soy y en el que me resuelvo, al menos parcialmente, nos abre a la experiencia de un sí que proyectando se proyecta, y que es asumido como una tarea; ciertamente, el sí que puede no es desechado, no dejo de hacer la experiencia propia de ser dado sin este anclaje en la estima de sí, vale decir en la creencia o convicción 
de que puedo; pero al mismo tiempo, el sí-mismo queda cada vez abierto a una posibilidad que lo saca de sí. Se podría afirmar que los posibles a los que nos abren las capacidades contestan el "yo soy" con un "yo puedo" que está lanzado y enviado siempre delante de sí. Claro, será preciso luego adentrarnos en una reflexión sobre la paciencia y el consentimiento, para recobrar en algún sentido al sí que somos ante la contestación del "poder-ser". En todo caso, se trata de una contestación y no de un rechazo.

4. Ricœur por otra parte, navega entre dos aguas, al momento de afirmar que el sí-mismo se atestigua y reconoce en las capacidades. Pues, ¿qué significa esto? ¿Acaso que éstas son un modo esencial y a priori de constitución del sí-mismo? Ciertamente, nuestro poder-ser es constitutivo de nuestra ipseidad de éxodo, pero al mismo tiempo es preciso afirmar que éstas no son un a priori, al menos que se pueda establecer un a priori relacional, lo que no es tan fácil mostrar (Cf. ROMANO, 2011a). Se puede decir, sin embargo, que las capacidades son una estructura del modo de ser del sí-mismo, pues Ricour se ha abocado a mostrar, desde Le volontaire et l'involontaire, que lo que somos es lo que podemos; pero al mismo tiempo es preciso afirmar que en nuestras capacidades nos arrostramos "lo que somos" primeramente como "lo que podemos", y por tanto como una posibilidad antes que como un hecho. Pero aún es preciso delimitar el cómo de este arrostramiento.

Ser capaz implica, como hemos dicho, una apertura hacia posibles que podemos apropiarnos; los poderes en los que nos atestiguamos nos lanzan a un mundo que nos aparece recorrible, manipulable, contemplable, etc., solo por los recursos que nos donan las capacidades en las que nos ejercitamos; así, el poder de caminar, por ejemplo, nos permite percibir una montaña como una tierra escalable; percibir aquella montaña es comprenderse en una posibilidad que se nos abre y que está fundada en nuestro poder-ser. Pero al mismo tiempo, atestiguarse en las capacidades es aventurarse hacia modos específicos de ser que no podían ser anticipados antes del encuentro al que ellas nos abren. No basta decir que somos capaces de actuar, de decir, de narrar y narrarse, de imputarse responsabilidad, pues cada poder se delinea conforme al modo cómo nos vuelve hacia el mundo y la relación a la que nos abre. Así, por ejemplo, si nos comprendemos como sujetos capaces de amar, no se puede, sin embargo, afirmar que sepamos cuál es el cómo de nuestro poder-ser sin haber hecho el encuentro con una persona amada. Es este último que nos arrostra nuestro poder-ser como el ser que estamos llamados a realizar y nos 
envía hacia un modelamiento o estilo específico de nuestros poderes. Así, si bien me afirmo en el poder de amar, me aventuro en los diversos modos cómo me comprometo como amante conforme a los encuentros que me demandan y me envían. El sí de las capacidades no está nunca delimitado, pues su apertura es relacional, pero también responsiva. ¿Por qué? Pues, ser capaz es, primeramente, ser capaz de responder a estos encuentros como se responde a desafíos que nos ponen en juego. Los poderes en los que nos atestiguamos son, por tanto, no solo modos de entrar en relación, sino respuestas en la que nos arrostramos nuestro ser como un poder-ser. La respuesta, como modo de ser del sí-mismo, aporta algo que la relación no puede, la implicancia. Si bien es cierto que solo un sujeto capaz de relación puede responder, es necesario también subrayar que la respuesta compromete al sujeto con aquello a lo que está abierto; supone un ser capaz de asumir las exigencias que nacen de los encuentros que modelan o estilizan, a su vez, al sí en sus capacidades. De este modo, "ser capaz" es poder responder de sí y de lo otro; pues, su movimiento intencional -atravesado por la alteridad que afecta y fatiga al sí-mismo- cobra sentido en la manera como se asumen los posibles hallados o proyectados en la aperturidad propia del "poder-ser": hablar de apropiación no es hablar meramente de relación, pues, tal como en el caso del horizonte perceptivo, se puede estar en relación con una multiplicidad de objetos sin que todos ellos sean parte de nuestra atención. Mientras que en la respuesta lo que hay, lo que acontece es un intento apropiativo, no de lo otro que exige o demanda el despliegue de nuestras capacidades, sino de sus exigencias y del poder mismo que es conminado. Es en este sentido que respondemos de sí y del otro, pues es uno mismo el llamado a atestiguarse en sus poderes y los posibles a los que nos abren, pero no podríamos hacer esto sin la intervención de la alteridad que nos apela. Es ésta, finalmente, la que nos hunde en un campo de exigencias del que no podemos sustraernos: el responder; pues ser capaz implica poder elegir nuestras respuestas, el modo como nos arrostraremos nuestro ser, pero supone también no poder no responder.

De este modo, lo propio del responder es poner en juego al sí-mismo que hace del reconocimiento de sí una dificultad fundamental. Así, por ejemplo, tal como nos lo recuerda Ricour, basta volver la mirada a Le volontaire et l'involontaire para corroborar toda la dificultad que nos plantea la decisión: un significar en vacío, un proyecto, un yo capaz de llevar a cabo lo decidido y que se reconoce como tal, un sujeto que acoge motivos y que transforma la necesidad en razones para actuar, etc. La decisión hace del reconocimiento de sí un reconocimiento difícil en cuanto ella misma está llena de alteridad y el 
sujeto que decide ve quebrada su autonomía en pos de una heteronomía: en la decisión soy por mí mismo, me resuelvo por mí mismo, pero también soy por otro.

La decisión me pone en relación con los poderes con los que cuento para ejecutar un proyecto, y en este sentido, "me" direcciona primordialmente hacia el futuro. Pero es preciso distinguir dos modos de volverse hacia el porvenir: el primero concierne a la previsión, el segundo al proyecto. La previsión implica una conciencia teórica capaz de establecer relaciones de causa y efecto, por ejemplo, en las que el sujeto que prevé no está puesto en juego, así por ejemplo puedo prever que mañana saldrá el sol o que hará buen tiempo, etc., sin que ninguna de estas afirmaciones me impliquen de ningún modo que no sea accidental. Por el contrario, el proyecto me designa como su responsable y ejecutor. Si el proyectar es un elemento esencial de la decisión, el tender hacia aquello "por hacer" me hace responsable de llevar a cabo la ejecución del pragma de mi decisión, del proyecto. Aquí se revela una responsabilidad singular que recae en el sujeto y que de algún modo le arrostra su ser en sus proyectos, en sus direccionamientos, en su volcarse de modo propio hacia el futuro. La decisión me arrostra una responsabilidad, pues implica un sujeto que puede aquello que proyecta, y que solo "él" lo puede.

La decisión no está lanzada hacia el futuro de manera lineal e irrevocable, sino elípticamente: "Es porque ese futuro enfocado no está restringido al orden continuo y reversible del tiempo vivido: de proyecto en proyecto salto más allá de los tiempos muertos; vuelvo a momentos anteriores; bosquejo los ejes más interesantes de la acción futura, encierro las lagunas, pongo fines delante de los medios que los preceden, inserto proyectos secundarios en proyectos primarios por engaño gradual o intercalación. Tal es el tipo de temporalidad que organiza el tiempo delante del presente. La discontinuidad y la reversibilidad, continúa Ricœur, son la ley de ese tiempo designado en vacío donde solo son significadas las relaciones prácticas de los plazos más notables de la acción (la duración misma de la acción proyectada puede ser por otra parte significada, por ejemplo, para otro, a título de enseñanza, de modelo a imitar, o de orden a ejecutar)" (RICCEUR, 1950, p. 48). La discontinuidad y reversibilidad suponen un sujeto capaz de retomar los proyectos cada vez de diferente manera conforme a los múltiples poderes con los que cuento. En este sentido, el sujeto que proyecta al mismo tiempo se proyecta de diversas formas siendo capaz de retomarse en los proyectos cada vez de nuevo. Pero, si el proyecto implica un sujeto que puede lo que quiere, que tenga el sentimiento 
de poder, por lo mismo implica también un sujeto que hace la experiencia de la incapacidad: no todas sus acciones, no todos sus poderes, son capaces de alcanzar lo proyectado. Proyectar es querer y poder lo que puedo, y en tanto tal implica también "no poder" todo lo que quiero y puedo.

Nada podría ser proyectado por un sujeto sin el sentimiento de que puede lo que proyecta y que lo lanza hacia lo querido. El sentimiento de poder o estima de sí (RICCEUR, 1990) me designa singularmente sin que nadie pueda ocupar mi lugar. $\mathrm{Al}$ respecto nuestro autor afirma que

[...] es preciso reintroducir aquí ese sentimiento de poder que acompaña el enfoque de la conciencia. Es ese sentimiento que reúne al yo proyectado como sujeto de la acción por hacer y al yo apercibido en sordina como aquel que proyecta. Yo que quiero, yo puedo. Yo que decido hacer, yo soy capaz de hacer; y es esta capacidad que yo proyecto en el sujeto de la acción. (RICEUR, 1950, p. 47).

El sentimiento de poder revela tanto el hecho de que "puedo" como al "yo" que puede sobre un fondo de impotencia insuperable. Pero, ¿qué impone restricciones al poder y al querer? La situación dada y el mundo al que nos abrimos con nuestros proyectos y nuestras acciones. "Yo alojo mis proyectos, afirma Ricœur, en los intersticios de un mundo determinado en sus grandes líneas por el curso de los astros, por el orden de todo" (RICCEUR, 1950, p. 49). Que los proyectos en los que nos comprendemos, reconocemos y atestiguamos, nos lancen al mundo, implica que nos abren nuevos posibles gracias al poder de iniciativa con que cuenta el hombre. Pero al mismo tiempo el sujeto se encuentra en el mundo con posibles que salen a su encuentro. Proyectar implica por tanto la capacidad de tener que reunir ambos posibles, los proyectados y los encontrados. Ciertamente los que salen a nuestro encuentro, siendo posibles, pueden ser también fuente de obstáculo para los proyectos en los que nos empeñamos ofreciéndonos razones para desviarnos parcial o definitivamente de aquellos. Podría decirse que aquellos posibles no proyectados tienen la fuerza de lanzarnos de otro modo y hacia otros fines, y por tanto de refigurar nuestra orientación en el mundo, así como pueden dar un mayor impulso a los proyectos en los que nos comprometemos: en suma, los posibles que vienen a nuestro encuentro pueden vulnerar la orientación que nuestras capacidades nos ofrecen; pero no podríamos hallar posibles no intencionados por nosotros sin contar con las capacidades suficientes para que éstos se hagan presentes para nosotros. 
5. Los posibles tienen su fundamento en nuestro cuerpo como fuente de poderes. "Lo posible adquiere una consistencia y como un espesor carnal; está en el camino de lo real; es la capacidad de la realización del proyecto por el cuerpo" (RICEUR, 1950, p. 53). Por que las capacidades tienen como fundamento el cuerpo como órgano del querer, como punto cero de nuestras perspectivas, como fuente de la espacialidad del mundo y como afección, debe afirmarse también que ellas se fatigan y son vulnerables. De este modo, si el sí-mismo se atestigua en su "poder-ser", al mismo tiempo se experiencia irremediablemente en la fatiga vivida como una impotencia, como un peso, como un límite para el despliegue del sí en sus capacidades. Ciertamente, el cansancio no es del cuerpo en estricto sentido, pues "soy yo quien se cansa" y no una materia orgánica cualquiera; pero, ese "yo" se fatiga porque es corporal, porque posee un cuerpo vivido (Leib) que es la fuente de la experiencia como una relación abierta al mundo y a sí. Cansarse, de este modo, no es solo desfallecer, sino que es hacer depender el aparecer del mundo estrictamente de aquel que ya no puede más. La fatiga no suprime el mundo, le da, más bien, un peso y consistencia que recaen sobre el sujeto que se esfuerza en resistir. Al respecto, Jean-Yves Lacoste afirma:

Hablar de mundo, excúsenme la trivialidad del recordatorio, es hablar del horizonte de todo aparecer. Y en la experiencia de la fatiga, es necesario concebir que todo me aparece diferentemente -lo que quiere decir que el mundo es pura y simplemente diferente. Estoy claramente fatigado de esto o aquello, de haber leído la Crítica de la razón pura o de haber hecho una larga excursión, pero la cosa fatigante, o la acción fatigante, tiene por notable propiedad fenomenológica el repercutir en toda otra aparición. La Crítica de la razón pura me fatiga. Pero, en el acto en que ella me fatiga (y en este acto solamente, claro - me será siempre posible hacer otra cosa y permitir al mundo aparecerme diferentemente), es todo el mundo, en bloque, y es aquí el punto importante, el que me fatiga. (LACOSTE, 2006, p. 311).

De este modo, la fatiga nos permite poner el acento en las siguientes consideraciones: en primer lugar, el cansancio pone en juego a un sí-mismo y no meramente a un cuerpo entre otros; como ya lo hemos afirmado "soy yo quien se cansa"; pero al mismo tiempo es necesario precisar que lo atestiguado no es un "yo", sino un "si", pues el sujeto que hace la experiencia de la fatiga se reconoce en una implicación estricta con la alteridad, viviéndose a sí mismo en la tensión de las resistencias del esfuerzo y de la fatiga. Que se trate de un sí y no meramente de un "yo", significa también que la fatiga aporta razones para 
desestimar las pretensiones de autofundación y de transparencia propias del ego cogito. El sí fatigado no solo mantiene una relación de implicación con lo otro de sí, siendo su propio cuerpo vivido otro para sí, sino que además responde de su cansancio oponiéndole las resistencias del esfuerzo o consintiendo la fatiga como signo de felicidad o de un gozo que se quisiera perpetuar. En este sentido, el cansancio que experienciamos no se traduce necesariamente a una experiencia de negatividad, sino que puede ser considerado bajo la luz de la afirmación originaria: un sí-mismo feliz de su cansancio es también un sí paciente que, lejos de querer persistir en ser el "mismo", prefiere volver productiva o feliz la relación con la alteridad. Así, si la fatiga parece volver nuestra mirada hacia atrás, hacia un sí-mismo cuyo proyectar ha sido interrumpido parcial o definitivamente por el cansancio, también nos vuelve hacia adelante, hacia un porvenir que es el propio de la paciencia. Pues, hay algunos cansancios que portan consigo un futuro, un posible, y una apertura; hay aquellas fatigas que se experiencian como una afirmación a la afectación en la que se reconoce el sujeto cansado. Mientras que hay cansancios que nos sustraen posibles, hay otros que nos los aportan.

Pero, si no todas nuestras fatigas nos lanzan a la tarea de tener que ser y apropiarse sus posibilidades, toda fatiga, sin embargo, se sostiene en la paciencia como modo de ser propio del sí-mismo. "La paciencia, afirma Emmanuel Housset, no es ni una pura pasividad dócil, ni la constancia de una voluntad, es un cierto modo de permanecer frente a lo que se da, que no es una simple permanencia en el tiempo de la sustancia, sino de durar manteniendo la relación" (HOSSET, 2008, p. 27). En un sentido semejante, Philippe Grosos afirma:

Ciertamente la paciencia es virtud, y pocos pueden disponer de ella. Sin embargo, antes de ser una virtud, y tal vez incluso para poder serlo y ser pensada como tal, debe encontrar su necesidad primera en lo que primeramente no depende del hombre: el tiempo y su duración propia. $Y$ en efecto, toda existencia pacienta, consiéntalo o no. Su paciencia, su inmemorial paciencia, es su temporalización misma, su despliegue, la extensión de su duración propia. Al considerar las cosas bajo este ángulo, la impaciencia no es entonces sino una posibilidad humana entre otras que no altera en nada a la originaria paciencia. Ser de un carácter impaciente no permite en efecto escapar a la paciencia que el despliegue de la existencia requiere (GROSOS, 2004, p. 8). 
Si la paciencia es el trasfondo de la fatiga, se puede decir que aquellas fatigas felices atestiguan al sí-mismo de modo prospectivo, pues lejos de replegarse en sí el sujeto cansado y abatido por las resistencias a su iniciativa, se atestigua, más bien, a partir de la afectación que provoca la fatiga; no se trata ni de un cansancio de sí, a partir del cual el sujeto ya no querría más consigo mismo, ni de una fatiga de lo otro que supondría una voluntad de acorazamiento; por el contrario, el sí-mismo que hace una experiencia feliz del cansancio persiste en la relación con lo otro como fuente de su cansancio, pues éste es vivido como una posibilidad de atestación de una identidad posible, futura, que se anuncia en la fatiga. Lejos de sustraerle posibilidades, lo llena de posibles que pueden ser asumidos. Este es el caso, por ejemplo, de la fatiga del sujeto que ora: no hay allí una resignación, un abatimiento desgraciado, sino una constancia que buscar seguir abierto ante lo otro, ante Dios, y que espera poder aún responder a su conminación.

6. El cansancio de la oración se sustenta, por tanto, en una espera: poder comprenderse por lo otro; así como mantenerse en vigilia constante, persistente: pero, ¿qué sería de la vigilia sin la experiencia de la fatiga? ¿No es acaso toda vigilia un modo de reponerse y de resistirse a la fatiga del día, de la jornada? Y al mismo tiempo, ¿no habría que afirmar que nunca se está en vigilia si ésta no cuenta ya con una fatiga que le acompańe y que jamás ceda del todo? O incluso, ¿̨no se puede afirmar que hay vigilias queridas, buscadas, que cuentan con la fatiga de la jornada como un modo de afirmarse, de afirmar la existencia en contra de la vida, reducida ésta a las funciones biológicas que obran en nosotros sin nosotros como en el caso del sueńo? Es así como Lacoste presenta esta cuestión:

El tiempo de la vigilia es verdaderamente nuestro tiempo, aquel que ganamos sobre la no-libertad y la no-conciencia, sobre las puras necesidades biológicas. Este tiempo, por lo mismo, no puede ser reivindicado frente a tribunal alguno. Privarme del sueño o del sueño necesario para una buena salud, sería un abuso: tengo derecho a que el Estado o la empresa, salvo en caso de emergencia, me dejen dormir. El acto de velar nos aparece, así, como pura posición de sí, como compendio de una afirmación de nuestra libertad. Y una vez terminados los trabajos cuya distribución nos es necesaria, previsible y ordinaria, una vez cumplidos los deberes diurnos, la decisión de velar prueba que nos queda un derecho capital: el de probar, por el contenido que damos a nuestra vigilia (que podemos ocupar para hacer filosofía, escribir poesía o rezar -y muchas otras cosas más), el plus de sentido que damos a nuestra humanidad. La vigilia no me revela a mí mismo tal como debo ser, sino tal como deseo ser. (LACOSTE, 2010, p. 119-120). 
La compleja relación entre fatiga y vigilia nos permite insistir en el hecho que la identidad del sujeto que hace su experiencia es en primer lugar prospectiva: pues la vigilia, tal como lo indica Lacoste en el texto recién citado, es un modo de apostar por una identidad querida, por un yo que puedo ser y que no se restringe ni a la experiencia actual de éste ni al modo de dárseme éste en la memoria retencional. Querer mantenerse despierto no es solo resistir al dominio de la vida por sobre la existencia humana, implica también una paciencia específica: la de la duración de sí. Interesante es reconocer que en la vigilia querida o buscada -a otras determinaciones se llegaría seguramente en el caso del insomnio tal como ha sido analizado por Levinas-, a pesar de la fatiga que la asecha, el sujeto se resuelve en ella como en una respuesta. Afirmar que la vigilia es la ocasión para reconocer y conferir ese "plus de sentido que damos a nuestra humanidad" no es otra cosa que reconocer una afirmación paciente: la de querer resolverse a sí-mismo por sí-mismo, en el fondo último de nuestras decisiones. En este sentido, se puede entender el velar como una "crisis" de sentido, como una crítica al "yo puedo" que quisiera detener su influjo en los hábitos adquiridos como meras disposiciones sedimentadas, antes de entenderlo como una disposición a nuevas posibilidades. Si estos argumentos tienen algo de sentido, entonces la vigilia es más que un mantenerse despierto, es una insistencia en ser ese sí-mismo que podemos y que aún no somos, que en cuanto lo podemos, en cierto respecto lo somos, pero nunca del todo ni de modo definitivo.

Por otra parte, lo que ponen en juego la fatiga y la vigilia, cada cual a su modo, es la paciencia de sí, aunque siempre de manera imbricada. Pues, finalmente el carácter prospectivo de sí, que se haya implicado en ambas experiencias, supone también la experiencia de la distancia temporal: la paciencia no siendo aquí la virtud del instante, sino la espera de la constancia y la insistencia. El sí fatigado, como el sí en vela, ambos están expuestos a una experiencia de retardo de sí, donde se encuentran a sí, no se dan a sí mismos sino a partir de un poder que me sitúa no delante de mí mismo, sino en un envío, en una tarea, en una posibilidad, lo que no es exactamente estar delante de sí. Se podría afirmar que el sujeto conminado más que encontrarse delante de sí, se haya perdido y sumido en una tarea que es preciso desentrañar, descubrir, pues su ser está primeramente resuelto en eso que llama, que conmina, que envía. Pacientarse a sí mismo implica por tanto asumir la tarea de tener que buscarse a partir de su pasibilidad, en las contingencias que hacen del sí un traumatismo, desgraciado o feliz. Pues, si la vigilia, el estar en vela es de algún modo un tipo de atención particular, una búsqueda o un hacer que busca, 
también se trata por ende de una experiencia expuesta al encuentro: encontrar, ser encontrado o encontrarse. Estar y mantenerse despierto, resistiendo los ritmos del sueńo y la vigilia vital, suponen una insistencia en la relación con el mundo y lo otro, una atención, aunque desfalleciente si se quiere, que prefiere la inquietud como modo de ser antes que un acorazamiento cualquiera. $\mathrm{He}$ aquí la paciencia de la vigilia: querer mantenerse abierta y alerta al mundo, persistir, insistir en ser en relación con éste y consigo mismo, en cuanto el sujeto en vela resuelve experienciarse en sus poderes (o en la fatiga de éstos), privilegiando el darse a sí mismo corporal, pues no tenemos noticia de nuestra cuerpo si no es por los poderes disponibles a partir de los que nos comprendemos como sujetos actuantes y sufrientes. Del mismo modo, hacer la experiencia del mundo es hacer la experiencia de que puedo alcanzarlo (en un sentido análogo, cf. HENRY, 1965, p. 133), intencionarlo, recorrerlo, etc., conforme a los poderes con que cuenta para hacerlo: son estas capacidades las que abriéndome al mundo, me dan su experiencia a partir de los posibles que las capacidades corporales pueden tomar, percibir, apropiarse, al tiempo que los poderes del sujeto son llamados a ser estilizados por las exigencias emanadas de la relación con el mundo percibido o experienciado. La vigilia es un modo de estar en el mundo que acentúa el privilegio que tiene el sí de existir conforme a sus capacidades y al libre despliegue en el mundo que ellas posibilitan. Ciertamente, un análisis como este requiere de una serie de precisiones que no daremos en este texto, pero que al menos podemos enunciar. No vale lo mismo para toda vigilia: esto ya lo hemos dicho cuando hemos insinuado una diferencia entre el insomnio, como un estar en vela a nuestro pesar, y la vigilia buscada o querida. En el primer caso, pareciera que no es posible sostener la insistencia del sujeto por privilegiar su existencia como modo de ser relacional y responsivo que distingue de la vida que ocurre pasivamente en nosotros; en el insomnio el hombre hace la experiencia de un il y a, de una neutralidad de la que no puede nada; en la vigilia buscada se trata más bien de ser en la paciencia de nuestro esfuerzo, de persistir en nuestra acción y en la comprensión de nosotros mismos a partir de nuestros poderes. Pero también se puede distinguir entre esta última vigilia y la del hombre que reza, que es a lo que se aboca realmente Jean-Yves Lacoste y Jean-Louis Chrétien (1999), mas sobre esto último no nos detendremos, no siendo la ocasión para comprometernos en este análisis. 
7. Entroncando con el último parágrafo podemos centrar nuestros an álisis en el "hombre capaz de escuchar y de leer" que prefigura la hermenéutica reflexiva que Ricœur desarrolló a partir de 1960 y que culmina, a nuestro juicio, con una filosofía de la llamada y la respuesta que fue elaborada de manera explícita en sus trabajos de hermenéutica bíblica y, de manera menos explícita pero no menos insistente, en su hermenéutica del sí-mismo. La idea de detenernos en esta cuestión, y de algún modo proponer una reconstrucción de la "llamada y la respuesta" al interior de la fenomenología del hombre capaz, es por el interés que tiene a doble título: en primer lugar, nos permitirá confirmar que la atestación de sí acontece en la alteridad, en la relación con el otro o lo otro, siendo el sí-mismo un sujeto enviado y conminado; en segundo lugar, esta es la ocasión para delinear de mejor manera qué es el responder como un modo de ser propio del sí-mismo; éste, espero, puede ser un lugar privilegiado para insistir en lo que se puede llamar "la paciencia de la respuesta", a partir de la que el sí-mismo se comprende en una identidad prospectiva.

Volvamos al punto de inicio de este trabajo: ¿qué es la reflexión? Según Ricœur, ésta es "[...] la apropiación de nuestro esfuerzo por existir y de nuestro deseo de ser, a través de las obras que testimonian este esfuerzo y este deseo" (RICEEUR, 1965, p. 56). Y un poco más adelante, agrega: “[...] la reflexión debe volverse interpretación, porque no puedo captar este acto de existir en otra parte que en los signos esparcidos en el mundo" (RICCEUR, 1965 , p. 57). Tal como hemos afirmado al comienzo de este trabajo, lo que Ricœur subraya con la reflexión es, por un lado, el itinerario intencional y de retorno de la vida subjetiva: el sí es aquel que estando lanzado hacia las cosas mismas cuentan con la posibilidad de retomarse en sus obras, en los signos que testimonian aún su esfuerzo por existir y deseo de ser. Detengámonos un momento en estas cuestiones. En primer lugar, qué se dice cuando se afirma que el hombre se esfuerza en existir. La idea de esfuerzo supone siempre una resistencia vivida y un movimiento del que somos capaces y nos sabemos capaces. Tal vez, sobre esta idea ricoeuriana, se haya ya un esbozo privilegiado del "hombre capaz", pues ¿en qué consiste este esfuerzo si no en comprenderse capaz de volcarse hacia el mundo según el modo de la libertad? Es decir, que la existencia requiera de nuestros esfuerzos no significa otra cosa que ésta es un modo de ser que podemos apropiarnos asumiendo el riesgo de perdernos en el mundo por nuestros poderes por los que nos lanzamos a él. Pero, un esfuerzo, como afirmábamos recién, supone una resistencia. Así, el volcarnos intencional hacia las cosas mismas implica también que el mundo, las situaciones, los acontecimientos, etc., se nos dan ya como una 
imposibilidad, como una resistencia a nuestra acción, a nuestro ser "expuesto", "existencial". Nuestro modo de ser "hacia" se confronta cada vez con una oposición, con un obstáculo que puede pausar momentánea o definitivamente nuestra orientación en el mundo. Pero, también se trata de un esfuerzo por que nuestro existir no solo requiere de nuestras capacidades a partir de las que atestiguamos ese "quién" que somos, sino también porque conlleva la exigencia de la obra, de obrar en el mundo y desplegar y comprender nuestra existencia en los signos que producimos. Lo que ofrece resistencia a nuestro esfuerzo es, en este punto, el carácter abierto y ambiguo del signo, lo que hace que nuestra inscripción en el mundo no sea nunca definitiva ni esté acabada. Es por ello que la reflexión tiene que volverse interpretación del mundo y del sí, explicitación del mundo y del sí.

Por otra parte, el deseo de ser, tal como es señalado por Ricœur, enfatiza dos dimensiones relevantes para el ser humano: en primer lugar, el hecho de que nuestra vida se deja determinar primariamente por el deseo y, por consiguiente, por el anhelo de orientarnos en el mundo en busca de un télos que da sentido a la unidad de nuestra vida. Hay aquí, ciertamente, un esfuerzo por priorizar las filosofías de la afirmación, de la vida, del sí en sus múltiples modos de decirse y darse. Pues, una vida deseosa es también una que apuesta por la posibilidad de alcanzar un sentido a partir del cual se trame. De este modo, de lo que se trata es de un deseo por el que el hombre deposita su confianza en el sentido como sentido buscado y alcanzable. En segundo lugar, el "deseo" de ser pone énfasis en la indeterminación o inconclusión radical de lo deseado y de todos los movimientos que debemos realizar para alcanzar su cumplimiento. Del mismo modo, el "deseo" de ser nos abre a la exigencia de interpretar y explicitar el mundo y al sí que vuelve hacia éste, de explicar más para comprender mejor. Lo que significa, a su vez, que el ser que somos nos es dado como una tarea y no como un dato o un hecho. Ese ser que estamos llamados a realizar, a cumplir, a llevar a cabo, no se deja comprender a través de una intuición inmediata, sino que se "está haciendo", está deviniendo en algo más, dándose a una interpretación continua. El deseo de ser nos indica por tanto que estamos llamados a ser algo que aún no somos del todo, que ese "quién" que somos es más bien del orden del acontecimiento y no del hecho: se "llega a ser", no se es del todo; lo que supone al mismo tiempo que el símismo está expuesto, por su modo de ser relacional y responsivo, a la fuerza impredecible de los acontecimientos, por ejemplo, biográficos, aquellos que irrumpiendo en la vida del sujeto provocan modificaciones profundas en el modo cómo éste se comprende y se orienta en el mundo. El sí-mismo se hace 
en los múltiples esfuerzos en los que se compromete como existente donde la vida intencional puede ser entendida como su paradigma, pero también "se" constituye al encuentro con lo que no se deja recibir sino a la postre, a saber el acontecimiento biográfico; allí, el sí no es el autor de "lo que le pasa", y sin embargo se recibe asumiendo una responsabilidad por aquello que "lo da a sí mismo" en una acogida más antigua que toda recepción, y que "le da" al mismo tiempo un envío: refigurar el sentido de su existencia, explicando más para comprenderse mejor. Lo que también puede implicar un "narrar más para comprenderse mejor".

La interpretación, en la que se ha vuelto la reflexión, es por tanto la forma que tiene el sí-mismo de retomarse a sí no en la forma de "lo que es", sino en su "haciéndose", pues a fin de cuentas lo que requiere interpretación es el sentido que se despliega delante como proyectado o lanzado allí. Que el sí deba retomarse en la interpretación de los signos, las obras, los símbolos, los textos, las acciones consideradas como un cuasi-texto, significa que lo que es interpretado es un sentido puesto delante de sí, por ejemplo delante de un texto, y que tiene la fuerza de conminar al interpretante, legándole una tarea constante, la de refigurarlo y refigurarse a sí mismo. De este modo, lo interpretado es más bien aquello a lo que soy conminado, la tarea a la que soy vuelto. Asumo una responsabilidad que excede a la responsabilidad por lo hecho, y toma la forma de una respuesta por lo que "debo ser". Ciertamente, se trata de una normatividad que debe ser integrada en la vida subjetiva, pero que tiene su fundamento en un deseo, y por tanto, en una carencia; se trata de un deseo ético en última instancia: reencontrarse en los actos, en los signos, en los símbolos, en los textos, en las acciones como cuasi-textos a través de la interpretación constante de la vida del ego vuelto hacia el mundo. A este deseo ético propio de la hermenéutica reflexiva, le seguirá otro, en el marco de la hermenéutica del sí-mismo, la del anhelo de vivir una vida buena con otros y para otros en instituciones justas. Sin embargo, esta segunda formulación debe quedar en suspenso para el cumplimiento de las intenciones primeras de este trabajo

Es preciso explicitar la relación entre la hermenéutica reflexiva y el "ser capaz de escuchar y de leer". Jean Greisch dice al respecto lo siguiente: "Leer y descifrar son actos tan distintos como deletrear y hablar. En el acto de leer, la legibilidad del texto está ya presupuesta, en lugar que ésta sea su resultado. Así como el auditor, el lector es el respondiente activo del texto que le hace reaccionar de un modo o de otro, suscitando por su parte palabras nuevas que pueden eventualmente ser consignadas en nuevos textos" (GREISCH, 
2006, p. 67). Ciertamente, tal como lo destaca el propio Greisch después de Ricœur, la lectura tiene un momento activo y otro pasivo: la recepción de un "[...] sentido preexistente depositado en el texto" (GREISCH, 2006, p. 67) y la apropiación de sentido en cuanto "[...] refiguración de sentido configurado por el texto" (GREISCH, 2006, p. 67). Pero esta dialéctica entre recepción y apropiación, que incluye por lo demás los diversos modos cómo puede ser entendida la escucha - como atención al sentido ya dado, que a su vez puede tomar la forma de la crítica y la sospecha, o como atención a las posibilidades libradas por el sentido del texto, sin ser ninguno de estos modos contradictorio entre sí, sino altamente complementarios-, remite a un modo de ser propio del sí-mismo: el de ser un respondiente. Pues, finalmente, leer y escuchar son capacidades por las que recibimos e innovamos un sentido, pero, tal como lo afirma Greisch, son también la ocasión de nuestras respuestas. Leyendo y escuchando asumimos la responsabilidad y el envío del sentido, asumiendo la tarea de desvelarlo y de cumplirlo. De esta manera, el sentido que es librado por un texto porta, él también, una conminación: la de responder de él, asumirlo en la innovación y la refiguración de nosotros mismos en él.

Pero, es preciso aún una última nota al respecto: el sentido del texto ante el que el sí-mismo se comprende no solo nos singulariza en nuestras respuestas, las múltiples refiguraciones en las que nos comprendemos, sino que también hace de nuestro mundo un mundo inteligible. Es en este sentido que Jean Greisch (2006, p. 69-70) puede afirmar que “[...] nosotros no 'leemos' solamente textos, sino que, de aproximación en aproximación, la experiencia como tal debe ser 'leída', para ser comprendida".

8. Los argumentos del parágrafo anterior nos permiten, a su vez, acentuar el movimiento centrífugo de la atestación: yo no me atestiguo ese "quién" que soy sino en las capacidades que son a su vez respuestas al encuentro con el otro y con lo otro. El hacer de la lectura y la escucha subrayan ejemplarmente cómo sí-mismo se reconoce en sus capacidades que solo toman forma o se estilizan singularmente al encuentro con los textos que libran un sentido por ser interrogado, pero que a la vez conmina al lector o auditor a refigurarse a sí mismo en relación a dicho sentido. Así, la dialéctica entre recepción y apropiación solo tiene lugar si el sentido que se da porta consigo un cierto envío o, si se quiere, una tarea por realizar. 
Ricœur asumirá también la tarea de llevar a cabo una reflexión pormenorizada sobre los modos cómo el sí-mismo se refigura en torno a los textos, particularmente bíblicos, que es lo que quisiera interrogar en este último parágrafo de este ensayo. La pregunta que se hace Ricœur en Amour et justice (2008), particularmente en los textos titulados "Le soi dans le miroir des écritures" y "Le soi mandaté. Oh my prophetic soul", es ¿en qué sentido el sí-mismo responde del sentido que viene dado en los textos? Lo que a su vez implica una tesis respecto del modo de ser respondiente o responsivo del sujeto. Pues, si el sí-mismo es tal porque está en relación con lo otro de sí su cuerpo, la conciencia, el otro, los textos, etc.-, significa esto que estar en relación es ya estar en posición de responder de aquello que adviene como sentido o sin sentido. La respuesta que damos es una toma de posición con respecto a lo otro y a sí mismo. Pero, más interesante aún, es que el responder no agota ni clausura la conminación a la que nos enfrenta el sentido. Es por ello, tal vez, que Ricœur le otorga un privilegio a la dupla "llamada-respuesta" por sobre la de la "pregunta-respuesta". Mientras que la segunda implica una determinación primera de la pregunta para avocarnos a la tarea de contestar a sus interrogantes, la llamada no se deja clausurar ni al lugar o emisor de donde proviene, ni tampoco se deja delimitar de modo definitivo, quedando su sentido conminante siempre abierto, dado a la interpretación y a sus conflictos. Responder a una llamada no es clausurarla, sino mantenerla abierta.

Es así que la respuesta a una llamada es, de alguna forma, una correspondencia, pues no se trata primeramente de aportar inteligibilidad a la llamada a través de nuestras respuestas, sino de corresponder a su envío, a sus intenciones, a su altura. El sí-mismo no solo escucha una conminación, entiende primero una conminación de la conminación, a saber la de interrogar su sentido y, a la vez, dejarse conducir por éste. La preocupación por el mandato de la llamada no quiere esclarecerlo de una vez y para siempre, pues interrogar su sentido es interrogarse a sí mismo con referencia a él. La conminación del llamado "bíblico", nos hunde en la inspección de ese "quién" que somos y que estamos convocados a convertirnos, un "quién", por tanto, una ipseidad que solo se resuelve en su ser si ella está verdaderamente en relación con lo otro de sí, con lo que llama y convoca. Aquello que el sí-mismo no lo es tan solo respecto de sí, sino primeramente en sus respuestas que intentan corresponder a las exigencias que vienen de la llamada del otro o de Dios.

Un sentido de la correspondencia lo aporta el propio Ricœur: 
Primero, yo no sitúo la respuesta frente a la pregunta, sino a la llamada. Y eso cambia todo: una cosa es responder a un pregunta, en el sentido de resolver un problema planteado; otra, responder a una llamada, en el sentido de corresponder a la concepción de la existencia que propone. Enseguida, esa llamada no viene de la filosofía sino que procede de la Palabra, recogida en las Escrituras y transmitida por la tradición y las tradiciones que resultan de esas Escrituras. Por último, la respuesta que tengo en vista no es la de la teología, en tanto que discurso más o menos sistemático, sino la del sí que, de sí llamado se vuelve en sí respondiente (RICEUR, 2008, p. 46).

$\mathrm{Al}$ menos se pueden aportar las siguientes aclaraciones: en primer lugar, la llamada no nos conmina a su desvelamiento, a menos que esto signifique que desvelarla sea al mismo tiempo "descubrirse", comprenderse en vistas del sentido y de la propuesta de mundo que ella libera cuando es escuchada o cuando es atendida por la lectura. En segundo lugar, la llamada nos ofrece un sentido habitable del que hay que responder. Esto supone que, por una parte, la correspondencia es un modo de dejarse implicar por la "concepción de la existencia que propone" [la llamada] (RICCEUR, 2008, p. 46) o, lo que es lo mismo, el sí que corresponde asume la tarea de comprenderse en relación a ese sentido de un mundo habitable que porta la llamada. Corresponder, entonces, es asumir la tarea de llevar a cabo ese sentido; se trata, ciertamente, de la performatividad de la llamada. Pero, por otra parte, corresponder a la llamada es un acto de confianza, hay allí una atestación quebrada; pues, de aquí en adelante me atestiguo capaz de llevar a cabo el sentido que la llamada porta y envía, con todas las fragilidades, vulnerabilidad y fatigas que implica la capacidad humana. Confiar en este envío es también reconocer que su sentido está expuesto a la contestación: nada asegura veritativamente que el sentido propuesto es adecuado, es concordante, etc., solo se puede confiar en ello. Es en este preciso momento que la atestación se deja decir también como testimonio; pues la correspondencia a la que está convocado el sí respondiente supone el ejercicio de sus capacidades como testimonios del sentido propuesto, de su sensatez; pero eso no significa que en el despliegue de mis capacidades no esté expuesto a la contestación del sentido que reivindico como propio. Así, el sí respondiente no está asegurado de antemano, no es un sí justificado, sino uno que apuesta por un modo de ser al cree corresponder, cuyo fundamento le escapa pero lo sostiene al mismo tiempo. No son las Escrituras la última palabra, pero sí la palabra a la que se busca responder. 
Ciertamente, se responde a otras muchas cosas, a otros llamados, como por ejemplo la interpelación de un hijo, de la conciencia, del otro como prójimo o del socius (cf. RICEEUR, 1967, p. 113-127) y de la institución. También se responde a las cosas mismas, a los símbolos y a los textos, donde responder no significa solo acoger pasivamente un sentido, sino innovarlo. No se podría corresponder a la proposición portada en la llamada de la fe bíblica, si las respuestas de las que somos capaces no fueran al mismo tiempo una refiguración, una innovación que delinea nuestra existencia en singular. No se responde de un mismo modo a la llamada, pues su conminación no supone su reiteración, su iteración, sino su reinvención. Aunque sea parte de su dimensión estructural que ésta sea iterable, la iteración no es su fin. Corresponder significa entonces, de algún modo, una asunción de su envío al mismo tiempo que una crítica de su sentido: no hay cómo corresponder a este último sin que asumirlo sea darle un estilo propio y singular que no responde nunca adecuadamente a la llamada, aunque busque hacerlo. En toda respuesta, incluso aquella que dice sí a la conminación, hay por tanto una refutación, no tanto de la llamada misma, sino de su arribo y del modo cómo puede ser apropiado su sentido.

Por otra parte, las capacidades, en su sentido estructural que es el primero que hemos intentado delimitar, ¿no son precisamente respuestas al encuentro con la alteridad que buscando apropiarse su sentido, se descubren conminadas a su realización? A su vez, una capacidad en sí misma no tiene sentido, pues ella se desarrolla, se educa y se despliega solo ante las exigencias y los envíos a los que lo otro la remiten. Solo porque hay amantes que nos conminan es que nuestra capacidad de amar toma forma y asume la ardua tarea de tener que corresponder a las exigencias y envío que vienen del otro. Responder es reconocerse y atestiguarse a sí mismo en el otro, más allá de sí. La atestación no es la instancia para que aflore el sí que somos por sí mismo, pues finalmente somos lo que somos solo en el otro, por sus envíos y exigencias. Atestiguarse es confiar que detrás de mis poderes hay un sí, un "quién” que, sin embargo, no se halla nunca de nuestro lado, sino siempre tendido en una prospectividad que es preciso asumir. 
MALET, Patricio Mena. Attestation and answer: reflection from a hermeneutic phenomenology of the capacities. Trans/Form/Ação, Marília, v. 36, n. 1, p. 137-164, Jan./ Abril, 2013.

\begin{abstract}
The following essay explores the thesis that in the attestation or recognition of the self, we attest to the self primarily in a prospective way. To give witness of oneself is not to recognize the self in oneself, but in a call, in an injunction that is the result of the encounter with the other. Thus we can affirm that personal identity is mainly an identity in exodus, that is, a sending identity. In order to analyze these theses I will focus on the hermeneutical phenomenology of the capable man that Ricœur developed explicitly in Soi-même comme un autre, and that is outlined in his work from Le volontaire et l'involontaire on. I will ask in what sense human capabilities are a way of being a respondent in the face of the other, and then, at the margins of the biblical hermeneutics of Ricœur, I will try to find the meaning that the act of responding has for the self.
\end{abstract}

KEYWORDS: Self. Attestation. Capacities. Call-Response. Sending.

\title{
REFERENCIAS
}

CHRETIEN, Jean-Louis. L'arche de la parole. Paris: PUF, 1999.

GENS, Jean-Claude. L'expérience vive. Paris: PUF, 2009.

GREISCH, Jean. Les multiples sens de l'expérience et l'idée de vérité. Recherches de science religieuse, tome 91, 2003/4.

GREISCH, Jean. Entendre d'une autre oreille. Les enjeux philosophiques de l'herméneutique biblique. Paris: Bayard, 2006.

GROSOS, Philippe. Linquiète patience. Chatou: La transparence, 2004.

HENRY, Michel. Philosophie et phénoménologie du corps. Paris: PUF, 1965.

HOUSSET, Emmanuel. La douceur de la patience. Revue d'éthique et de théologie morale, $\mathrm{N}^{\circ} 250,2008$.

LACOSTE, Jean-Yves. Présence et parousie. Genève: Ad Solem, 2006.

LACOSTE, Jean-Yves. Experiencia y absoluto. Salamanca: Sígueme, 2010.

LACOSTE, Jean-Yves. Etre en danger. Paris: Cerf, 2011.

MALDINEY, Henri. Penser l'homme et la folie. Grenoble: Million, 2007.

MARCEL, Gabriel y Ricœur, Paul. Entretiens. Paris: Aubier Montaigne, 1968.

MARION, Jean-Luc. Étant donné. Paris: PUF, 2005. 
MENA, Patricio. Homo capax. Teología y vida, Vol. LII, N 4, p. 675-693, 2011, IV Trimestre, 2011.

POREE, Jérôme. Justifier philosophiquement l'espérance. In: Paul Ricour: un philosophe lit la Bible. À l'entrecroisement des herméneutiques philosophique et biblique. Genève: Labor et Fides, 2011.

RICCEUR, Paul. Philosophie de la volonté. Tome 1: Le volontaire et l'involontaire. Paris: Aubier, 1950.

RICEUR, Paul. De l'interprétation. Essai sur Freud. Paris: Seuil, 1965.

RICEUR, Paul. Histoire et vérité. Paris: Seuil, 1967.

RICEUR, Paul. La foi soupçonnée. Recherches et Débats 19, nº 73, 1971.

RICEUR, Paul. La Révélation: discussion d'ensemble. In: La Révélation. Bruxelles: Facultés Universitaires Saint Louis, 1977.

RICEUUR, Paul. Temps et récit 1. Paris: Seuil, 1983.

RICCUR, Paul. Du texte à l'action. Paris: Seuil, 1986.

RICEUR, Paul. Regards sur l'écriture. La naissance du texte. Paris: José Corti, 1989.

RICCUR, Paul. Soi-même comme un autre. Paris: Seuil, 1990.

RICEEUR, Paul (1991). Un entretien avec Paul Ricœur. Soi-même comme un autre, (propos recueillis par G. Jarczyk). Rue Descartes. Revue du Collège International de Philosophie, 1991.

RICEUR, Paul. Parcours de la reconnaissance. Trois études. Paris: Stock, 2004.

RICCEUR, Paul. Amour et justice. Paris: Poche, 2008.

RODRÍGUEZ, Ramón. Hermenéutica y subjetividad. Madrid: Trotta, 2010.

ROMANO, Claude. Au cœur de la raison: la phénoménologie. Paris: Gallimard, 2011a.

ROMANO, Claude. L'aventure temporelle. Paris: PUF, $2011 \mathrm{~b}$.

Recebido em: 07.05.2012

Aceito em: 13.06.2012 
MALET, P. A. M. 